\title{
STRATEGIC CREW PLANNING TOOL IN RAILROAD: A DISCRETE EVENT SIMULATION
}

\author{
Kiran Chahar \\ Clark Cheng \\ Yudi Pranoto \\ Norfolk Southern Corporation \\ 1200 Peachtree St. NE \\ Atlanta, GA 30309, USA
}

\begin{abstract}
Norfolk Southern (NS) has developed a strategic crew planning tool to evaluate the impacts of crew rules changes and train service changes on crew utilization and train on-time performance. This tool has three major components, a discrete event simulator, a crew deadheading engine, and a crew pool size analyzer. A Flash based animation and reporting user interface helps users identify bottlenecks in specific areas of the rail network. This tool is integrated into a suite of planning tools used in NS. The impact of crew mark off rates on train performance is discussed in a case study.
\end{abstract}

\section{INTRODUCTION}

Freight trains are generally operated by an engineer and a conductor. The engineers and the conductors belong to specific crew pools depending on the territories and on the types of crew pools. Every crew pool has at least one home terminal and most crew pools have one or many away from home terminals. Figure 1 below represents the crew operation between terminals. Home terminal means that the crew has a home in that city and there is no lodging cost at Home. Away-from-home terminal implies that the crew needs a lodging arrangement for resting in that city. Home and away-from-home terminals have different business rules, for example, a crew waiting excessively at an away from home terminal can claim for detention payment while a crew at home cannot claim for detention. Each crew pool has a set number of jobs, called turns. Each turn is filled by one crew through a job bidding process where qualified crew with highest seniority level will get the turn. The turns form a queue on a crew board, generally made up of turns of the same pool to wait for their assignment to the trains. When the train arrives at its destination, the crew will tie up at another crew board according to the crew tie up rules. After at least 10 hours of rest, the crew will be available for his/her next assignment. Above mentioned freight train operations are modeled in our strategic crew planning tool.

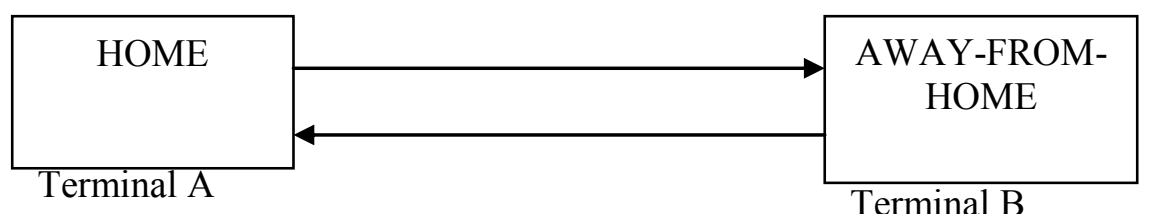

Figure 1: Representation of the crew operation

Optimization and simulation techniques have been utilized to model train crew assignment problem. Crew assignment problem in European railroad has been mathematically modeled by splitting this problem into two sub-problems of crew scheduling and crew rostering (Caprara et. al 1997). Freight train crew 


\section{Chahar, Cheng, and Pranoto}

operation of a North American railroad has been mathematically modeled as a multi-commodity network flow problem (Vaidyanathan et. al. 2007). Discrete event simulation modeling has been attempted to evaluate the impact on crew work schedule (Dalal 2001). Canadian National railroad in North America have developed its own simulation models for crew assignment process and presented their work in 2003 winter simulation conference (Guttukum et. al. 2003).

The complexity of crew business rules, various types of crew pools and their schedules makes it difficult to model it mathematically, as an optimization model may not capture the completeness of this process. A simulation model can capture the real operations and complex scenarios (Banks 1998). Discrete event simulation has been used to evaluate the real eventual effects of the changes on a real world without actually executing those changes on the real system (Law and Kelton 2003). Thus, NS resolved to use discrete event simulation to develop a strategic crew planning tool to include all the complexity involved in the real operations due to crew business rules for regulating crew assignment process. Few of these business rules common to all North American freight railroads are listed below:

- Pool Rotation: Every crew pool maintains a queue for placing turns. Then turns are called for jobs depending on their position in the queue and their availability (rested or not). Pool rotation is rules for maintaining this queue, for example:

- Arrival Time: Turns are placed in the queue according to train's arrival time at a terminal. i.e. turns arriving on early trains get to placed on top of the queue.

- Tie-up Time: It is the off-duty time of a turn. This is different than the train arrival time as turn has to finish some paperwork and other responsibilities after train arrives at a terminal. In this rule turns are placed according to their tie-up time, i.e. turn with early tie-up time gets prior positions in the queue.

- Last on-duty time: Turns are placed in a queue according to its most recent on-duty time. i.e. turn reporting early on-duty time gets to be placed on top of the queue.

- Crew tie-up process: When train arrives at a terminal, then turn finishes up some necessary work and reports its off-duty time as tie-up time. The terminal and the crew pool where turn needs to be tied-up are again decided by pre-negotiated union rules. For example, a turn can start from a home terminal and depending on the tie-up rule it can either have to come back to home terminal for tie-up or can tie-up in any specified pool located at train arrival terminal.

- Carded job schedules: These are crew pools with turns having specific schedules assigned to specific trains. These turns also have their assigned time window between which turns can work known as call window. Some turns also have schedules like a turn can take only first train or second train departing in the call window. A train can arrive anytime in the call window and the respective assigned turn will be notified to report on-duty for that train.

- Show up jobs: Generally all crews are contacted in advance by crew call system to report for the job. However, there are few crew pools in which crews have to report for the job at the specified time of the day without receiving any notification from crew call system. These crews have their fixed work schedules, like show-up on-duty everyday at 0830 hours.

- Rest rules: Every crew should be completely rested before showing up for the job. Every crew should get 10-hours undisturbed rest upon completing each job. In addition to this regular rest, crews are entitled for long rest periods too. For example, if a crew operates a train everyday for 7 consecutive days then it needs to rest for next 3 days.

- Mark-off rule: Crews can decide to take some vacation. This is done at the tie-up time at the home terminal only. At this point crew can decide to hold its turn for entire mark-off time i.e. no other crew can bid for this turn. Or crew can decide not to hold its turn and let other crews bid for the turn and fill it.

- Make-up turn rule: If turns from the assigned crew pool are unavailable then turns from back-up pool, also known as extra-board, are called for the job. These make-up turns are fictitious turns and are created for only one time job. It can be created and terminated only at the home terminal of a crew pool. 
- Detention rule: When a turn reaches its away-from-home terminal, it takes rest and is ready to head back to its home terminal. However, if there is no train to take, the turn might have to wait longer at away-from-home terminal. If a turn is there for more than some pre-specified number of hours, then that crew is entitled to be paid extra per minute. This is called crew detention.

- Maximum service quota: Every crew is assigned maximum number of hours it can be on-duty in a month. If a crew reaches its monthly quota then it has to take long rest till next month starts.

The idea of Norfolk Southern (NS) for developing the tool for crew assignment process differs from the one proposed by Canadian National in use of technology and development approach. This tool is integrated into a suite of planning tools used in NS. Being part of the integrated planning tools, it allows the flexibility of taking into the changes made in the train plan or crew plans from other planning tools itself without any delay or difficulty. The strategic crew planning tool by NS is unique in its capability of not limiting to study only the existing train plans in the system. User can also create its own train and crew plan using this tool. Other than the wide variety of output reports, it also provides an animated visual effect of the running simulation to assess the crew pool status and the train status simultaneously as simulation progresses over time.

In sections 2 and 3 we introduce the architecture and components of the strategic crew planning tool. In these sections it is explained how discrete event simulation is used in the train-crew assignment simulation and deadhead simulation. Section 4 briefly describes the output of this tool. Section 5 concludes this paper by presenting a case study and summarizing the importance of this tool for any railroad.

\section{SYSTEM ARCHITECTURE}

\subsection{Architecture}

We discuss the inputs and the outputs of the strategic crew planning tool in this section also presented by Figure 2. Input data module models variations in train departures, terminal dwell times, run times, crew rules, cost parameters, etc. The simulation module generates train arrivals and crew availability using the input data. A business rule engine matches crews to trains according to the pre-defined rules. An internal deadheading simulation module purports to mimic deadheading decisions to provide realism to the assignment process.

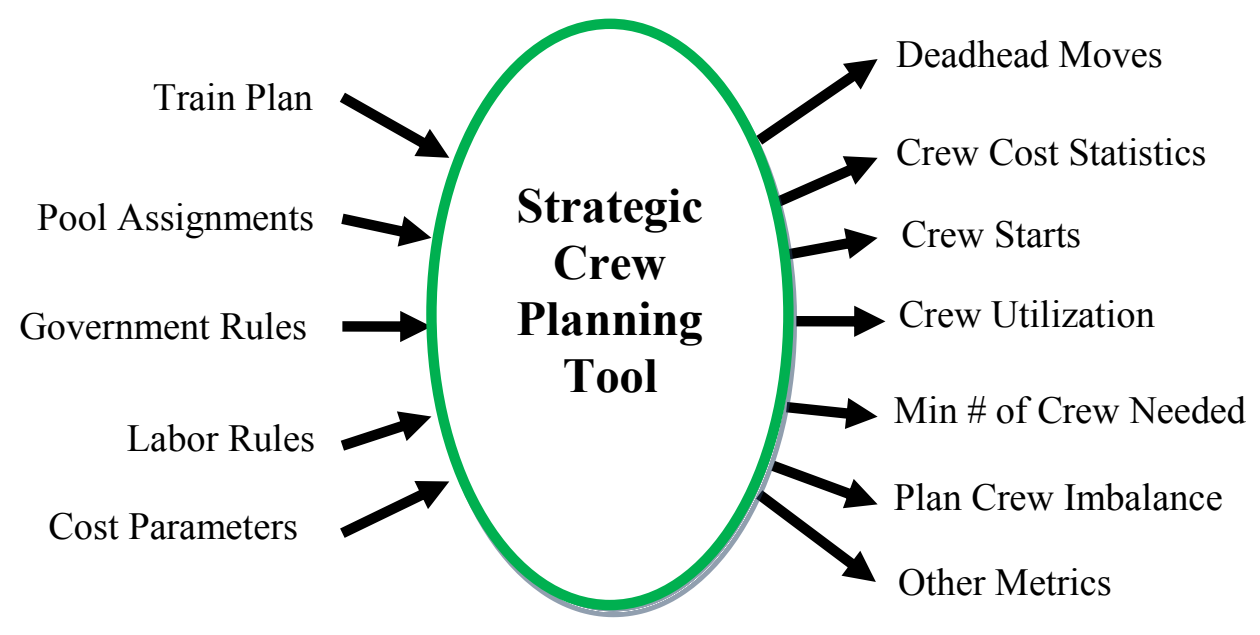

Figure 2: Architecture of strategic crew planning tool 


\section{$2.2 \quad$ Input}

\subsubsection{Train Schedule}

Train schedule is an input to the simulation engine. The input module of strategic crew planning tool is capable of creating the train schedule using historical data as well as current and future data on train plans. This set of trains is termed as 'Study'. 'Study' generation process creates a train operating plan between any start date and an end date. The 'Study' is created with arrival and departure times, and arrival and departure locations for each train.

\subsubsection{Crew Schedule}

Crew rules and regulations are also an input to the simulation engine. Rest rules for each crew pool, crew work schedule and call windows for carded pools are part of the crew plan. Crew plan also has the information on total number of crews in each pool, and the business rules for operating each crew pool.

\subsubsection{Business Rule Engine Assigning Crews to Trains}

All business rules to assign a crew to run a train in the study are modeled inside business rule engine. A business rule dictating which crew needs to run a train is referred as crew profile. This part of the module goes through a decision tree consisting of hierarchy of rules and assigns the best crew profile needed to operate the train. If a train cannot be assigned a crew profile in this process then user has to manually assign it. Once crew profile assignment process is completed then the train schedule becomes a complete 'Study' and is set for starting the simulation.

\subsection{Process}

\subsubsection{Simulation}

This simulation tool is developed using DESMO-J's discrete event simulation. In models of this type, all system state changes are supposed to happen at discrete points in time. Between such events the system state is assumed to remain constant. DESMO-J is implemented in Java. The usefulness of a web based interface (client-server architecture) for discrete event simulation has been studied and advocated (Whitman et. al 1998). This tool has client-server architecture and stores the simulation model on a main server so that potential users may access it freely and at the same time if needed.

This module takes train schedule (study) and crew operating plan as an input then simulates the crew to train assignments, train arrivals and departures, takes inputs from deadhead simulation and then incorporates the deadhead assignments into the simulation events to complete the process. The model makes several runs using the same core input data, and the summary statistics is generated from the average over the runs. In statistics collection process warm-up period statistics is removed to avoid its skewed effect.

\subsubsection{Deadhead Simulation}

Deadhead is the repositioning of crews between terminals either to balance the crew flow, or to prevent crew detention. Sometimes crews are deadheaded to a terminal to immediately take a train and spin around, this is called deadhead combo. It is not possible to deadhead combo from away-from-home terminal. Deadhead simulation engine considers all these complexities and runs inside the main simulation engine. The deadhead engine simulates the availability of crews (supply) and the train departures (demand) at each terminal (location). Based on the relationship of supply and demand deadhead engine makes it decision to deadhead or not to deadhead the crew and passes this decision on to the main simula- 


\section{Chahar, Cheng, and Pranoto}

tion engine in a form of which crew (name of the crew) needs to be deadheaded to which location (name of terminal) at what time.

\subsubsection{Pool Evaluator}

It happens that normal traffic is diverted from one location to other locations, and then it needs to be ensured that there are sufficient crews in the locations receiving the extra trains to meet desired service levels. This tool also has the option to let simulation engine suggest how many crews are required in each pool to achieve a target service level. In this option simulation engine goes through a series of decisions of adding or deleting the turns to achieve the target key performance indicator (KPI) value. This part is known as pool evaluator. The pool evaluator requires running multiple iterations and the number of iterations simulation needs to run before reporting the output is an input for this pool evaluator. In First iteration simulation decides to make changes to the crew pool standing and then again evaluates its decision before starting second iteration. For example, if it is specified that no more than 10 crews can be added in a complete simulation. Given this, at the end of first iteration pool evaluator evaluates its decision of adding and deleting turns and feeds its suggestion to the next iteration by ensuring that no more than 10 turns were added and the process continues till the end of last iteration run. As an output report various KPIs are evaluated for all iterations. User has the ability to analyze the results for all iterations and pick the crew plan from best iteration for crew hiring model.

\section{SYSTEM COMPONENTS}

\subsection{Crew Plan Parameters}

Crew schedules are the crew plan parameters for the simulation. Assignments for pools may change weekly or bi-weekly: it is not uncommon to delete or add turns to pools, depending on business levels. If crew members on average have not made their minimum number of miles, then turns may be deleted from a pool. If they will exceed a maximum number of miles, then turns may be added. Thus, this tool provides the flexibility for changing crew plan using various editors for changing number of turns in the crew pools, terminals for pool set-up, home and away-from-home terminals, carded job schedules, carded job call windows, show up pool schedules, crew profiles and adding or deleting extra-boards. The impact of these changes can be evaluated on various performance indicators like number of deadheads, train delays, crew utilization, crew availability, etc by running various simulation scenarios.

\subsection{Train Schedule Parameters}

'Train Viewer' is a convenient feature provided to view and edit the information on the train schedule parameters. Arrival time, departure time or the transit time for any train can be changed. The train associated crew profiles and crew terminal for any train can also be edited. This unique feature also has the ability to add new trains or delete existing trains from the already created 'Study'. This helps in fine tuning the train schedule as per the requirements. The before and after changes in the train schedule can be evaluated by comparing KPIs across different simulation scenarios.

\subsection{Crew Rule Parameters}

Crew rest rules and policies are changed very often without knowing their actual effect on the real system. For example, there were some crew policies and rest rule changes by FRA in July 2009. At these times there's a necessity to evaluate the impact of new changes and perform a comparison against the incumbent by means of performing the cost analysis and visualizing the trade-offs. This feature of the model allows to change the crew rest rules like mandatory rest hours at each terminal (home and away-fromhome), nature of rest (undisturbed or disturbed), eligible monthly service hours, and long rest rules. One of the important parameter is to specify the 'Mark-off Rate'. Mark-off rate can be either enabled or dis- 


\section{Chahar, Cheng, and Pranoto}

abled. In advanced options either one mark-off rate can be specified for entire system or pool specific mark-off rate can be specified. Model has a further capability of taking mark-off rate based on the day-ofweek as an input. Comparison of various reports like crew start, deadheading, train delay, crew activity, etc across all the simulation scenarios helps in analyzing the impact of mark-off rate and various rest rules on the system.

\subsection{Cost Parameters}

Every policy is associated with a cost to the system. Cost parameters like train operating cost, crew operating cost, deadhead cost, re-crew cost, and detention cost can be varied inside the model. It helps in performing the cost analysis and studying the trade-offs required to achieve the objective function.

\subsection{Diagnostic Reports}

Once a study is created this tool generates following diagnostic reports to perform a sanity check on the train schedule before starting the simulation.

\subsubsection{Crew Balancing}

This report helps in diagnosing the effect of business rule engine assigning crew profile to a train. Crew balance can be reviewed at each location by looking at number of inbound and outbound trains from a location. It can be easily verified whether a correct crew location has been assigned for the corresponding train location.

\subsubsection{Transit Time}

This report on transit time diagnosis helps in verifying that all the trains have transit times within the corporate regulated operating times for the crews. For example, a crew can be on-duty for the maximum of continuous 12 hours. After 12 hours crew has to immediately stop working and take 10 hours undisturbed rest. In this report if trains can be observed with transit time more than 12 hours implies that those trains are planned to have two crew assignments (re-crew situation). This process results in train delay and additional crew costs.

\subsubsection{Long Pool vs. Short Pool}

All pool set ups on given line segments can be viewed. Sometimes either due to train plan or due to labor union rules it is necessary to consolidate two or more pools or split a pool into two or more pools. For example, if there's a line segment from terminal A to terminal $\mathrm{B}$ and terminal $\mathrm{B}$ to terminal $\mathrm{C}$ and the transit time from terminal $\mathrm{A}$ to terminal $\mathrm{C}$ is within operational requirements then a pool from $\mathrm{A}$ to $\mathrm{C}$ can be combined and is known as long pool and the pool from A to B is known as short pool. With this diagnostics report user has the ability to view all the eligible trains and pools and make changes in train plan and crew plans before hand and evaluate various scenarios after running the simulation.

\subsection{Disruption Editor}

Railway track maintenance work is an always ongoing procedure and those tracks are completely shut down. Maintenance is based on pre-decided schedules for repairing tracks. The shutdown of tracks for maintenance work is called curfew. During curfew time, trains are re-routed using alternate line segments and alternate crew pools. Also, unforeseen service disruption can be caused due to any natural calamity. In unforeseen situations, particular region or area gets shutdown and trains will be re-routed. In such situations, with new train routes and new crew rules the new crew pools are also required. Simulation engine 


\section{Chahar, Cheng, and Pranoto}

has the ability to read in the disruption schedule and accordingly adjust the train plan and simulate the effects of any unforeseen service disruption or curfew disruption beforehand.

\subsection{Simulation Preview Mode and Replay Mode}

Simulation preview mode is provided to ensure that train and crew plans are set-up correctly before actually running the entire simulation. An animated mode of simulation with various running reports over time can be viewed on the NS system map in the preview mode. A sample of the reports produced during animation is presented in Figure 3. The various reports/charts presented are count of crews available in each pool at each terminal, status of trains (delayed, departed, arrived), status of crews, etc at each terminal. This is provided to visualize the bottleneck in the train plan or the crew plan. For example, if interested in a particular area then map can be zoomed into that area and look at the number of trains getting delayed and the corresponding crew pool standing at that time of simulation. All these insights helps in deciding if a train plan and a crew plan are consistent and provide a balanced schedule. Replay mode is the animated simulation version after simulation run has been completed providing an opportunity to look at the bottlenecks in detail at a later time.

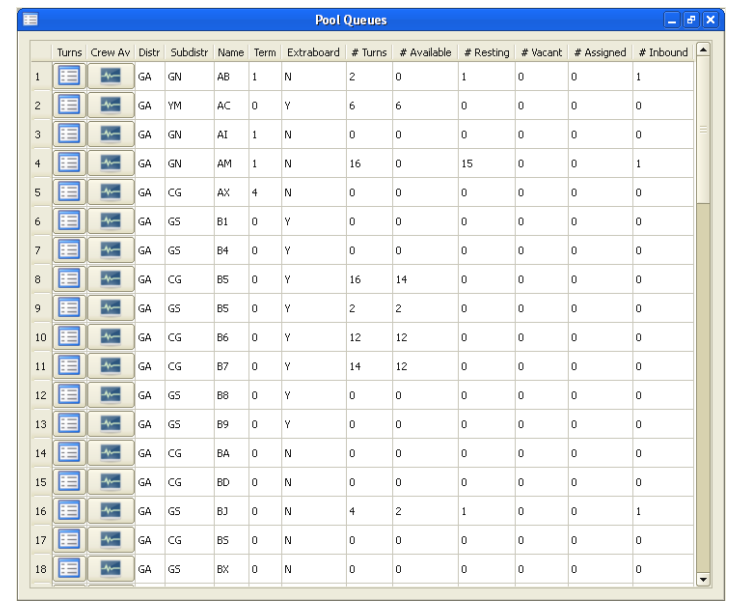

a. List of crew pools at a crew location

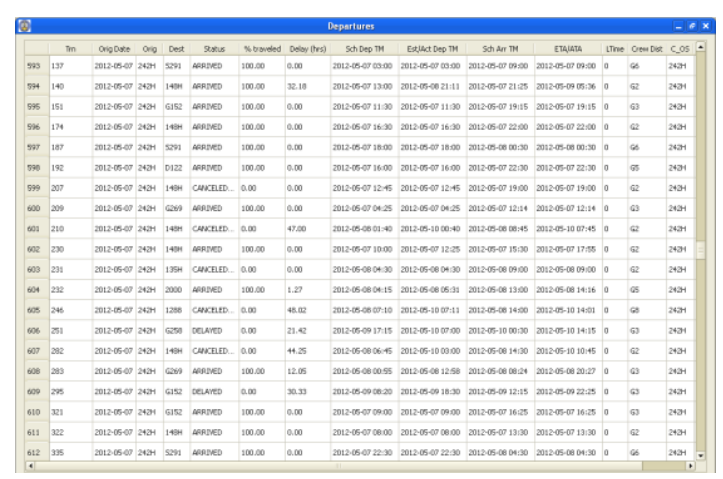

c. Status of originating trains

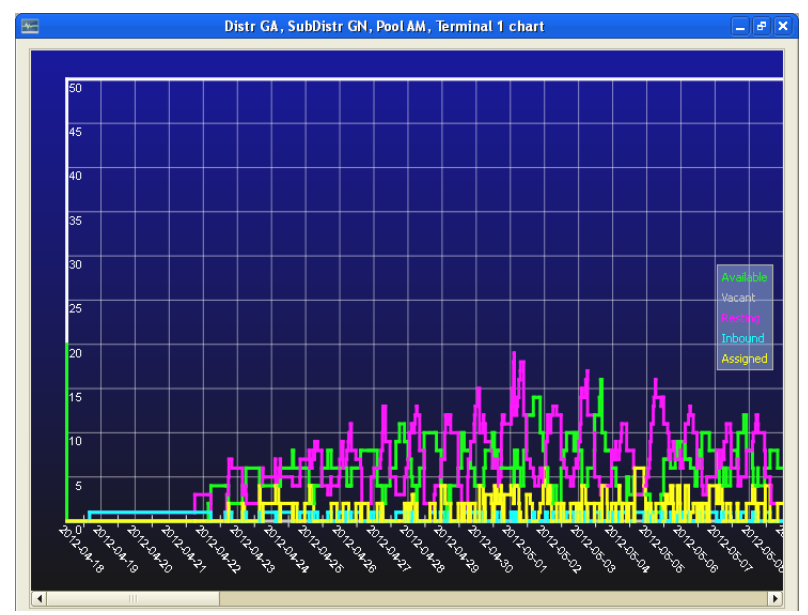

b. Crew pool standing over time

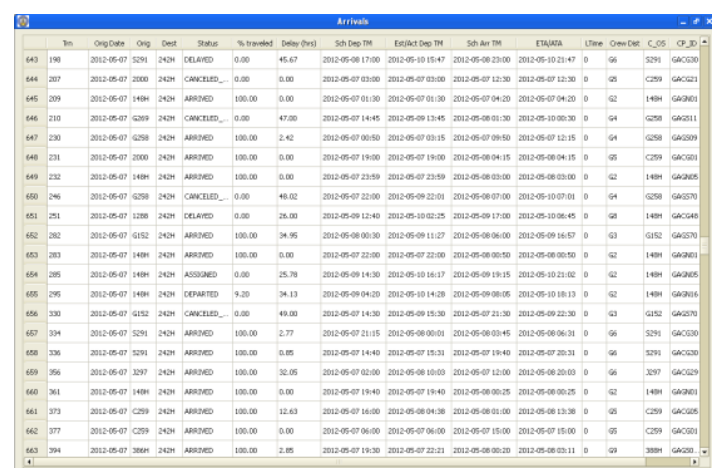

d. Status of terminating trains

Figure 3: Crew simulation preview

\section{SYSTEM OUTPUTS}

Various statistics is collected at the end of each simulation. Output is in the form of single simulation scenario reports and comparison reports among different simulation scenarios. 


\subsection{Single Simulation Output Reports}

\subsubsection{System Overview}

This report summarizes information on important KPIs on one page for a quick overview analysis. Various KPIs reported are: average number of hours a train is delayed, average train delay rate, average utilization rate of crew pools, average crew utilization further reported for carded job services and all other services, rate of deadheading, deadhead rate is also reported separately for carded job services and all other services and re-crew rate.

\subsubsection{Crew and Train Start Reports}

Starts are defined by three types: regular or normal start, deadhead start and re-crew start. Crew start report provides number of crew starts in a simulation. Train start report provides information on all types of train starts. The information in train start report also helps to analyze the utilization rate of each pool by observing the available size of pool versus turns actually used to operate trains in selected time period.

\subsubsection{Deadheading Report}

This report categorizes various types of deadheading in the simulation and presents numbers of each type of deadheads for each crew pool. Various reasons for deadhead decisions are identified as different types of deadheading as deadhead to balance pools, deadhead to prevent detention, deadhead to prevent excessive detention, and deadhead home before applying long rest rules.

\subsubsection{Crew Activity Report}

This is a report on average crew utilization rate. Utilization rate is defined as the productive time of the crew i.e. the percent of time spent by a crew in actually operating the train. This also presents detailed information on the percent of time spent in various states (like on-duty, resting, waiting for the assignment, etc) by a crew during the whole scenario.

\subsubsection{Train Delay Report}

It is a report on trains delayed in the simulation. This is presented on NS system map as delay rate or delay duration. Delays are color coded depending on the amount of delay. Further train delay can be viewed at division level (collection of similar crew line segments in terms of geographical location and labor rule agreements) or crew line segment level. Train delay is further divided into various delay buckets like delay due to unavailability of a crew, delay due to prior segment of the train or delay due to transit time.

\subsubsection{Crew Availability Report}

This presents the number of crews in various states (available, assigned, resting, inbound and onvacation) for each crew pool at any selected terminal at any given time of the simulation period. One of the most important benefits is to observe how a pool standing looks like at any point of simulation. It helps in analyzing if there's a need to add more turns or delete few turns depending on the availability or resting status of turns. If availability goes down immediately after simulation starts and remains like that throughout the simulation implies that there's a need of adding few more turns to the pool. As this downward trend of available crews implies that there are many more train starts (more demand) for the given pool as compared to current number of crews (less supply). 


\subsubsection{Train Statistics Report}

This is a detailed report with the data presenting what happened to each train in the simulation. It tells a complete story about a train in the simulation like, when it departed, when it arrived, from where it departed, who (name of crew) actually took the train, who was supposed to take the train, status of a train (like waiting for a crew, cancelled due to unavailability of a crew, arrived on time, or delayed), how many assignments were attempted to get a crew, and etc.

\subsection{Scenario Comparison Reports}

Simulation scenario comparison report is provided as a convenient feature to compare base and test scenarios and evaluate the various performance indicators. All reports in simulation comparison have the excel export utility. User can easily evaluate the impact of changing the simulation settings from one simulation run to another simulation run. For example, user might have made some changes in a train schedule and kept the crew plan unchanged and then evaluate the effect on crew availability, train delays, crew utilization and costs of changing the train schedule. Or user might keep the train plan unchanged but may change the crew plan by either changing rest rules or crew schedule or number of turns in the pool and then see the impact of changes by comparing the two simulation comparison reports.

\section{CASE STUDY AND CONCLUSION}

\subsection{Case Study}

The strategic crew planning tool developed by NS is able to answer the business questions always come up while designing a new train schedule or when new crew regulations are imposed. Following is a case study performed using the strategic crew planning tool at NS evaluating the impact of varying mark-off rate on number of number of train arrivals, deadhead rate, and train delays.

When starting the simulation various parameters can be specified in input module of the tool. One of the parameter is to specify the 'Mark-off Rate. Mark-off rate can be specified as one rate for entire system or different mark-off rates for each crew pool or further specify mark-off rate based on a day-of-week. A set of train 'Study' has been created from historical data (April - June 2010) and used the crew plan of June 2010 to run the simulation. In base scenario there is no randomness in train transit times, system mark-off rate is disabled i.e. mark-off rate is set to zero, normal crew rest rules of 10 hours undisturbed rest at home and away-from-home terminals are used. In test scenarios we kept everything same but varied the system wide crew mark-off rate. The chart in Figure 4 is an example showing the impact of only varying mark-off rate on the number of train arrivals, average train delay and total deadheads in the simulation run.

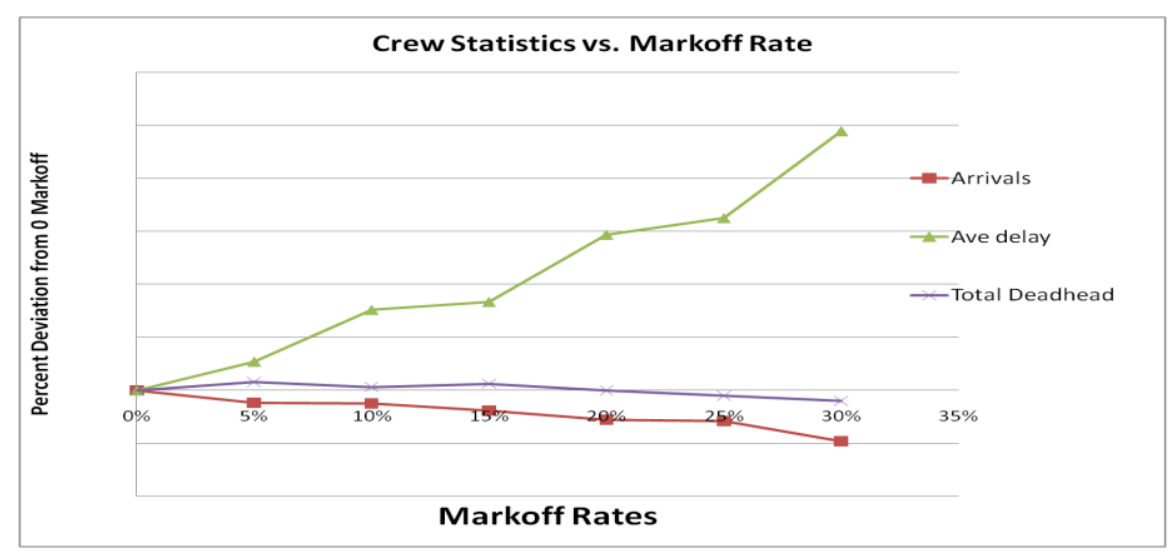

Figure 4: Effect of varying mark-off rate 1 
The chart in Figure 5 further breaks down the number of deadheads into various types of deadheads like: deadhead to balance pool standing, deadhead to avoid detention, deadhead of make-up turn return to home terminal and deadhead to home terminal before start of long rest rule.

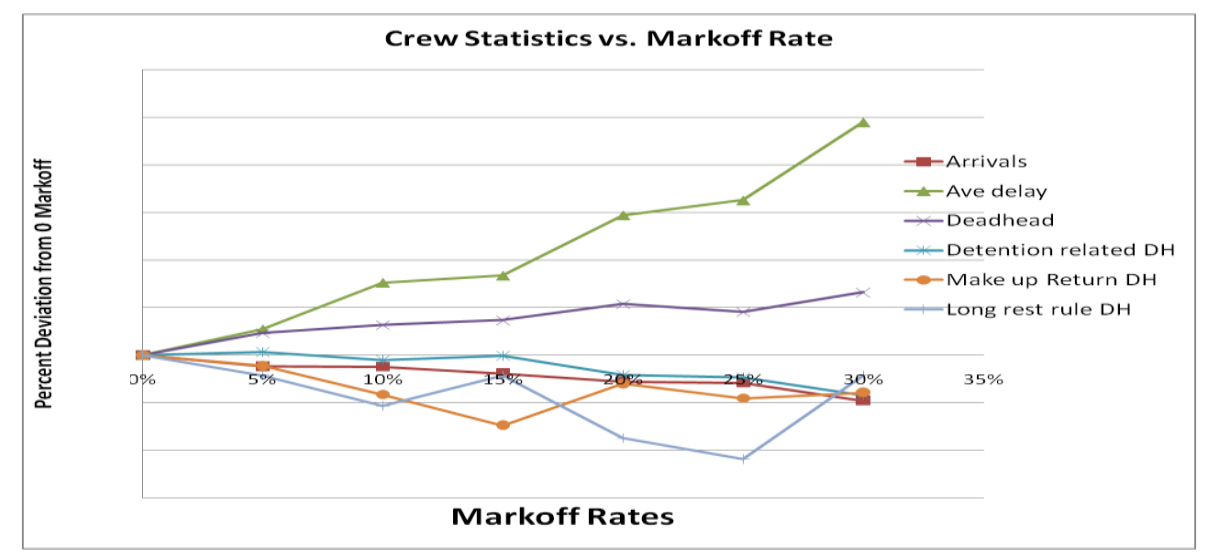

Figure 5: Effect of varying mark-off rate 2

Each mark-off rate percent represents statistics collected from the corresponding test simulation run as compared to the base simulation run. For example, in the test simulation as mark-off rate increases then the average train delay also increases and number of train arrivals decreases as compared to the base simulation run. It helps in understanding that as crew mark-off rate increases (like during holiday times) then more train delays and eventually more trains cancellations happen due to the unavailability of crews. Thus crew management has to prepare for holidays in advance to avoid such train delays or cancellations.

\subsection{Conclusion}

In our summary we conclude that we have presented the architecture, feature and few capabilities of the strategic crew planning tool developed by NS. The tool presented in this paper can evaluate the impact on crew utilization and costs when there is a need to change the schedule or routing of a train. If curfew is imposed on a given segment, this tool helps to see the impact on train delays, deadheading costs and crew utilization. This tool can evaluate all of following scenarios: how will a proposed curfew affect the availability of crews in the 48 hours that follow the curfew, if trains are rerouted or are just intentionally delayed then how does that affect the number of crews required in districts through which the train travels, if more trains would be added to the system then how would this affect deadhead costs on a given district? From the crew operation perspective this tool is able to evaluate the effect if a pool is added or deleted at a location, to evaluate the effect if crews are added or deleted in a crew pool, labor rules for one pool/one district are changed then evaluate the effect on crew utilization, train service metrics, and various costs. It further helps in analyzing the impact if home or away-from-home terminals (and rules) for one or more pools are changed, perform cost analysis when deadhead or detention costs and parameters (like the number of hours held away before detention claims kick in) are changed. Incomplete rest and inappropriate crew schedules results in crew fatigue and jeopardize safety and family life of the crew. User can utilize this tool to assess the effect of crew rules changes on crew fatigue and crew rest times by analyzing crew utilization reports. This simulation tool provides the ability to consider all above mentioned what-if scenarios which may prevent the adoption of policies resulting in increased operating costs to NS. 


\section{REFERENCES}

Banks, J., J. S. Carson, B. L. Nelson, and D. M. Nicol. 2000. Discrete-Event System Simulation. 3rd ed. Upper Saddle River, New Jersey: Prentice-Hall, Inc.

Caprara A., Fischetti M., Toth P., Vigo D., and Guida P. 1997. "Algorithms for Railway Crew Management." Mathematical Programming, 79 (1997), 125- 141.

Dalal M., and Jensen L. 2001. "Simulation modeling at union pacific railroad." In Proceedings of the 2001 Winter Simulation Conference, edited by B. A. Peters, J. S. Smith, D. J. Medeiros, and M. W. Rohrer,1048- 1055. Piscataway, New Jersey: Institute of Electrical and Electronics Engineers, Inc.

Guttkuhn R., Dawson T., Walker J., and Moroz M. 2003. "A Discrete Event Simulation for the Crew Assignment Process in North American Freight Railroads." In Proceedings of the 2003 Winter Simulation Conference, edited by S. E. Chick, P. J. Sanchez, D. M. Ferrin, and D. J. Morrice, 1686- 1692. Piscataway, New Jersey: Institute of Electrical and Electronics Engineers, Inc.

Law M., and Kelton W. 2003. Simulation Modeling and Analysis, The McGraw-Hill Companies, Inc.

Vaidyanathan B., Jha K., and Ahuja R. 2007. "Multicommodity Network Flow Approach to the Railroad Crew-Scheduling Problem." IBM Journal of Research and Development 51 (2007), 325- 344

Whitman L., Huff B., and Palaniswamy S. 1998. "Commercial Simulation Over the Web." In Proceedings of the 1998 Winter Simulation Conference, edited by D. J. Medeiros, E. F. Watson, J. S. Carson, and M. S. Manivannan, 335- 339. Piscataway, New Jersey: Institute of Electrical and Electronics Engineers, Inc.

\section{AUTHOR BIOGRAPHIES}

KIRAN CHAHAR is an Operations Research analyst at Norfolk Southern Corporation. She has been working in the railroad industry for 3 years and has participated in developing and implementing computer models and decision support systems for crew assignment and service design. She received her Ph.D. degree in industrial engineering from Clemson University. Her email address is <kiran.chahar@nscorp.com>.

CLARK CHENG is the director of the Operations Research group at Norfolk Southern Corporation, a Class I railroad in the U.S. He has been working in the railroad industry for 17 years and has participated in developing and implementing computer models and decision support systems for locomotive dispatching, car scheduling, network optimization, fleet planning, demand forecasting, yard simulation, crew assignments, service design, and car distribution. He received his Ph.D. degree in industrial engineering from Clemson University. His email address is <clark.cheng@nscorp.com>.

YUDI PRANOTO is the manager of the Operations Research group at Norfolk Southern Corporation, a Class I railroad in the U.S. He has been working in the railroad industry for 7 years and has participated in developing and implementing computer models and decision support systems for empty car distribution, crew assignments, and service design. He received his Ph.D. degree in industrial engineering from Georgia Tech. His email address is<yudi.pranoto@nscorp.com>. 